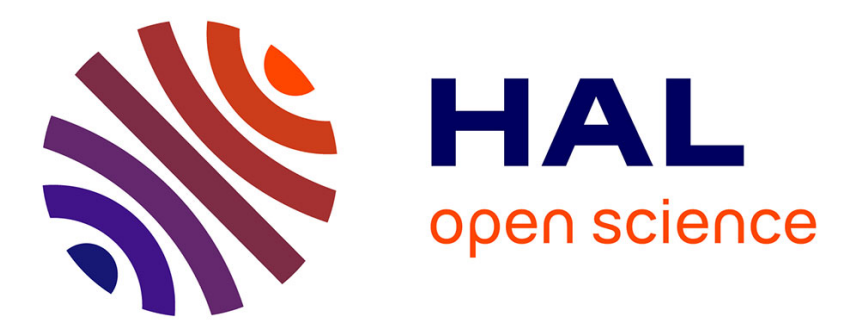

\title{
Influence de la non-stoechiométrie sur les propriétés ferroélectriques, de conduction et de relaxation de céramiques de type Sr6Ti2Nb8O30
}

\author{
A. Abalhassain, Jean Ravez, Jean-Pierre Doumerc, M. Elaatmani
}

\section{To cite this version:}

A. Abalhassain, Jean Ravez, Jean-Pierre Doumerc, M. Elaatmani. Influence de la non-stoechiométrie sur les propriétés ferroélectriques, de conduction et de relaxation de céramiques de type Sr6Ti2Nb8O30. Journal de Physique III, 1996, 6 (7), pp.863-872. 10.1051/jp3:1996160 jpa-00249497

\section{HAL Id: jpa-00249497 https://hal.science/jpa-00249497}

Submitted on 1 Jan 1996

HAL is a multi-disciplinary open access archive for the deposit and dissemination of scientific research documents, whether they are published or not. The documents may come from teaching and research institutions in France or abroad, or from public or private research centers.
L'archive ouverte pluridisciplinaire HAL, est destinée au dépôt et à la diffusion de documents scientifiques de niveau recherche, publiés ou non, émanant des établissements d'enseignement et de recherche français ou étrangers, des laboratoires publics ou privés. 


\title{
Influence de la non-stœchiométrie sur les propriétés ferroélectriques, de conduction et de relaxation de céramiques de type $\mathrm{Sr}_{6} \mathrm{Ti}_{2} \mathrm{Nb}_{8} \mathrm{O}_{30}$
}

\author{
A. Abalhassain $\left({ }^{1}\right)$, J. Ravez $\left({ }^{2, *}\right)$, J.P. Doumerc $\left({ }^{2}\right)$ et M. Elaatmani $\left({ }^{1}\right)$ \\ ( $\left.{ }^{1}\right)$ Laboratoire de Chimie du Solide Minéral, Faculté des Sciences Semlalia. \\ Université Cadi Ayyad, Marrakech, Maroc \\ $\left({ }^{2}\right)$ Institut de Chimie de la Matière Condensée de Bordeaux, Château Brivazac, \\ Avenue du Docteur A. Schweitzer, 33608 Pessac Cedex, France
}

(Reçu le 10 Juillet 1995, révisé 21 février 1996, accepté le 5 avril 1996)

PACS.77.80.-e - Ferroelectricity and antiferroelectricity

\begin{abstract}
Résumé. - Des céramiques de $\mathrm{Sr}_{6} \mathrm{Ti}_{2} \mathrm{Nb}_{8} \mathrm{O}_{30}$ ont subi des traitements de recuit sous atmosphère oxydante ou réductrice. Les matériaux cristallisent avec la structure bronze quadratique de tungstène et présentent une transition ferroélectrique-paraélectrique. Il existe une relaxation de charges d'espace à basse température pour les échantillons frittés à l'air. Un tel comportement disparaît après un long recuit soit sous oxygène (les grains et les joints de grains sont alors isolants), soit sous hydrogène-argon (les céramiques deviennent alors semi-conductrices extrinsèques de type $\mathrm{n}$ ).
\end{abstract}

\begin{abstract}
High densified $\mathrm{Sr}_{6} \mathrm{Ti}_{2} \mathrm{Nb}_{8} \mathrm{O}_{30}$ ceramics have been annealed under either oxidizing or reducing atmosphere. The materials crystallize with a tetragonal tungsten bronze structure and show a ferroelectric-paraelectric transition. A space charge dielectric relaxation occurs at low temperature for air sintered samples. Such a behavior disappears after long annealing under either oxygen (grains and grain boundaries are thus insulators) or hydrogen-argon (the ceramics become extrinsic n-type semiconductor).
\end{abstract}

\section{Introduction}

Les composés de formule chimique $\mathrm{A}_{6}^{2+} \mathrm{M}_{2}^{4+} \mathrm{Nb}_{8} \mathrm{O}_{30}(\mathrm{~A}=\mathrm{Sr}, \mathrm{Ba}, \mathrm{Ca}, \mathrm{M}=\mathrm{Sn}, \mathrm{Zr}, \mathrm{Ti})$ sont de type "bronzes quadratiques de tungstène" et présentent une transition ferroélectriqueparaélectrique. Les auteurs antérieurs signalaient que $\mathrm{Sr}_{6} \mathrm{Ti}_{2} \mathrm{Nb}_{8} \mathrm{O}_{30}$ cristallise dans le système orthorhombique ( $\mathrm{mm} 2)$ ou quadratique $(4 \mathrm{~mm})$ et qu'il présente une transition ferroélectriqueparaélectrique respectivement à 373 ou $333 \mathrm{~K}[1,2]$. La présence de $\mathrm{Ti}^{4+}$ dans ces composés favorise leurs performances diélectriques [2-5].

Dans ce travail nous avons étudié ces composés en réalisant plusieurs traitements thermiques sous atmosphère oxydante (oxygène) ou réductrice (mélange argon/hydrogène, dans le rapport 90-10) dans le but d'établir une corrélation entre les propriétés ferroélectriques, de conduction et de relaxation diélectrique.

$\left(^{*}\right)$ Auteur auquel doit être adressée la correspondance (Fax: (33) 56842761 ). 


\section{1. Élaboration des céramiques}

Le composé $\mathrm{Sr}_{6} \mathrm{Ti}_{2} \mathrm{Nb}_{8} \mathrm{O}_{30}$ est préparé par réaction à l'état solide à partir d'un mélange stœechiométrique de $\mathrm{SrCO}_{3}, \mathrm{TiO}_{2}$ et $\mathrm{Nb}_{2} \mathrm{O}_{5}$, chauffé à $1000^{\circ} \mathrm{C}$ pendant 15 heures. La poudre blanche obtenue est broyée pendant 1 heure puis mise sous forme de disques de $9 \mathrm{~mm}$ de diamètre et de $1,5 \mathrm{~mm}$ d'épaisseur environ. Les pastilles obtenues subissent différents traitements thermiques avec des vitesses de chauffe et de refroidissement de $250^{\circ} \mathrm{C} /$ heure en atmosphère oxydante ou réductrice (Tab. I).

\section{Analyse radiocristallographique à $300 \mathrm{~K}$}

Des études par diffraction des rayons $\mathrm{X}$ effectuées sur la surface circulaire des céramiques montrent que ces dernières sont constituées d'une phase unique de type "bronzes quadratiques de tungstène". La figure 1 montre les diffractogrammes des céramiques (a), (d) et (e) à $300 \mathrm{~K}$, ceux des céramiques (a) et (d) s'indexent dans le système quadratique avec les paramètres cristallins $a=12,360 \pm 0,005 \AA$ et $c=3,870 \pm 0,002 \AA$. Les nouvelles réflexions qui apparaissent pour la céramique (e) impliquent une distorsion vraisemblablement orthorhombique.

\section{Analyse par microscopie électronique à balayage}

Les céramiques étudiées sont cassées en deux demi-pastilles ; la surface de la fracture est métallisée par évaporation sous vide, puis observée au microscope électronique à balayage à des grossissements allant de $\times 500$ à $\times 20000$. Les micrographies représentées sur la figure 2 correspondent à un grossissement $\times 5000$. Cette analyse a permis de comparer les différentes microstructures obtenues et de déterminer en particulier la taille des grains ainsi que leur répartition en taille. Les caractéristiques microstructurales des céramiques frittées sont reportées au tableau II.

\section{Mesures diélectriques}

Les mesures diélectriques sont réalisées de 77 à $500 \mathrm{~K}$, sous hélium sec à l'aide d'un pont de capacité automatique Wayne-Kerr B905 aux fréquences $10^{2}, 10^{3}$ et $10^{4} \mathrm{~Hz}$. Les figures 3 et 4 montrent l'évolution thermique de la permittivité $\varepsilon_{\mathrm{r}}^{\prime}$. La céramique (a) frittée à l'air présente,

Tableau I. - Caractéristiques des céramiques de $\mathrm{Sr}_{6} \mathrm{Ti}_{2} \mathrm{Nb}_{8} \mathrm{O}_{30}$.

[Characteristics of $\mathrm{Sr}_{6} \mathrm{Ti}_{2} \mathrm{Nb}_{8} \mathrm{O}_{30}$ ceramics.]

\begin{tabular}{|c|c|c|c|c|c|}
\hline $\begin{array}{c}\text { Type de } \\
\text { céramique }\end{array}$ & $\begin{array}{c}\text { Conditions } \\
\text { de Frittage }\end{array}$ & $\begin{array}{c}\text { Traitement thermique } \\
\text { après frittage }\end{array}$ & Compacité & $\begin{array}{c}\text { Retrait } \\
(\%)\end{array}$ & Couleur \\
\hline (a) & air & & 0,93 & 17 & jaune \\
(b) & $\mathrm{O}_{2}$ & & 0,86 & 15 & blanc \\
(c) & $\mathrm{O}_{2}$ & & 0,91 & 16 & jaune-clair \\
(d) & $\mathrm{O}_{2}$ & 4 jours à $850^{\circ} \mathrm{C}$ puis & 0,86 & 15 & blanc \\
& & 4 jours à $600^{\circ} \mathrm{C}$ sous $\mathrm{O}_{2}$ & & & \\
(e) & $\mathrm{Ar} / \mathrm{H}_{2}(90 / 10)$ & & 0,92 & 17 & noir \\
\hline
\end{tabular}

(*) Température : $1250{ }^{\circ} \mathrm{C}$; durée : 2 heures. 


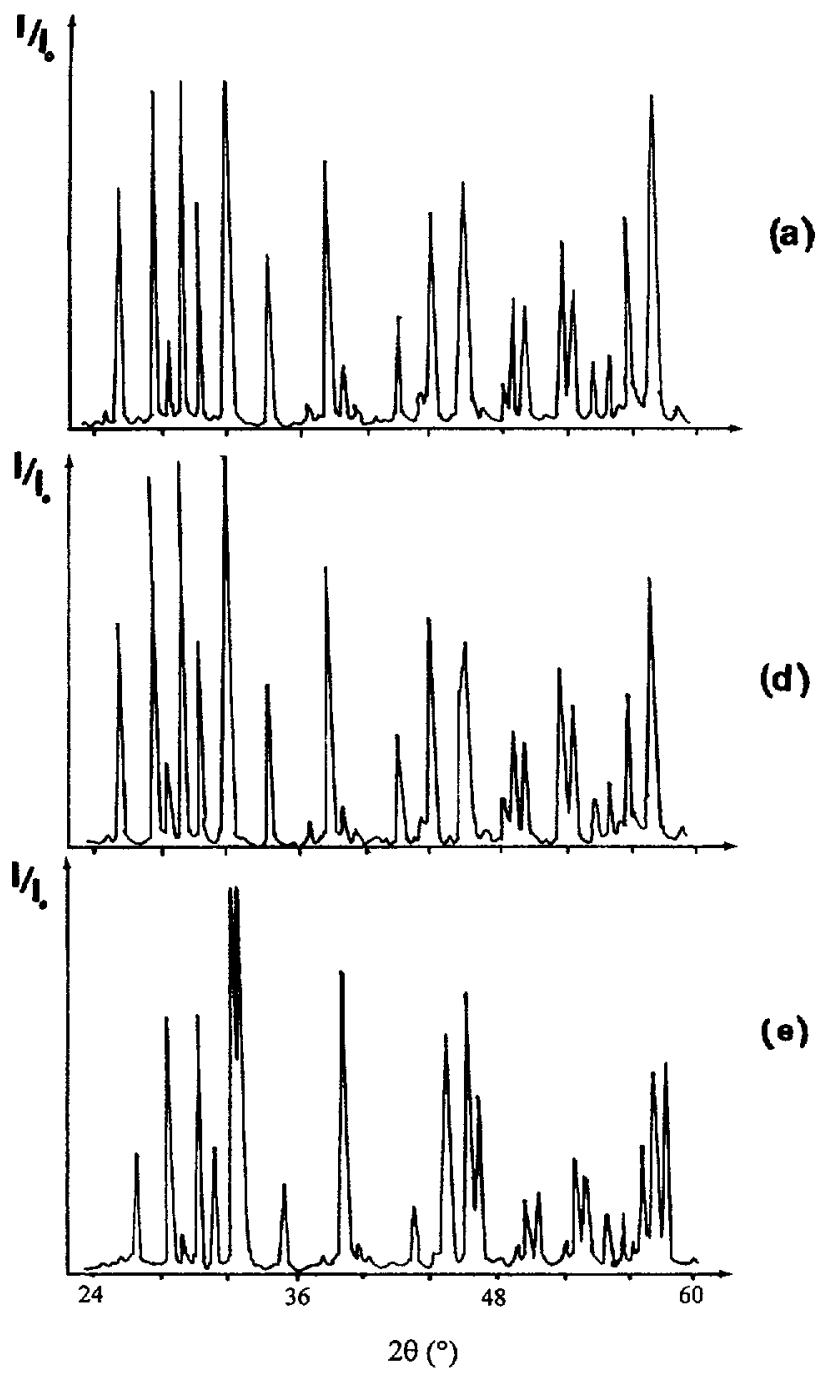

Fig. 1. - Diffractogrammes des céramiques (a), (d) et (e) à $300 \mathrm{~K}$.

[X-ray diffraction patterns of (a), (d) and (e) ceramic samples.]

à basse fréquence et à basse température (100 à $200 \mathrm{~K})$, une dispersion en fréquence caractéristique d'une relaxation : l'intensité du maximum de $\varepsilon_{\mathrm{r}}^{\prime}$ diminue et sa température augmente quand la fréquence croît. Ce phénomène diminue pour des céramiques frittées sous oxygène (b et c) et disparaît pratiquement pour la céramique (d) qui a subit un recuit prolongé sous oxygène. Le traitement sous atmosphère réductrice provoque la disparition du phénomène de relaxation (e). L'anomalie ou le pic observé à plus haute température (300 à $450 \mathrm{~K}$ ) et correspondant à la température de Curie, évolue également avec le type de traitement thermique. Les valeurs de la température de Curie déterminées à partir des mesures diélectriques sont rassemblées dans le tableau III. 


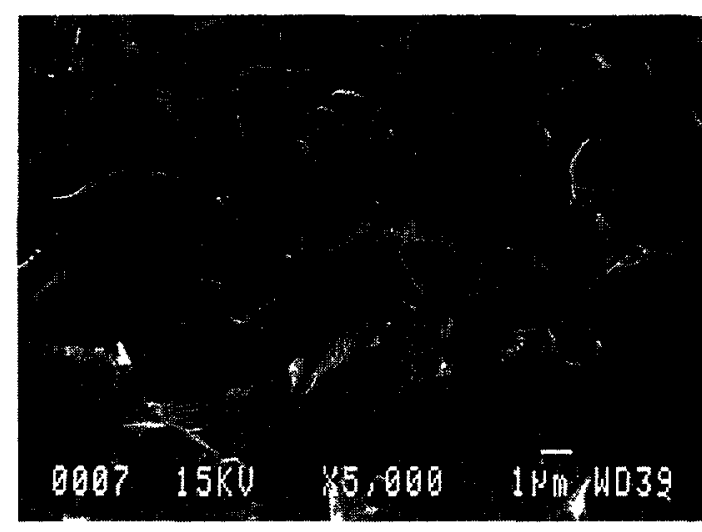

(a)

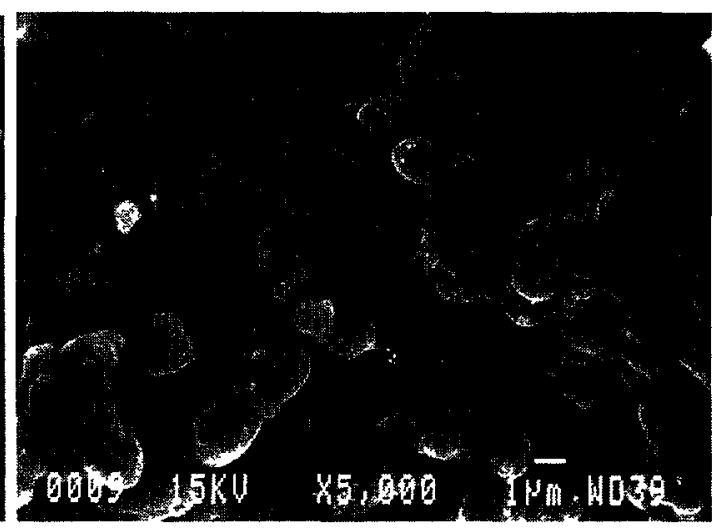

(b)

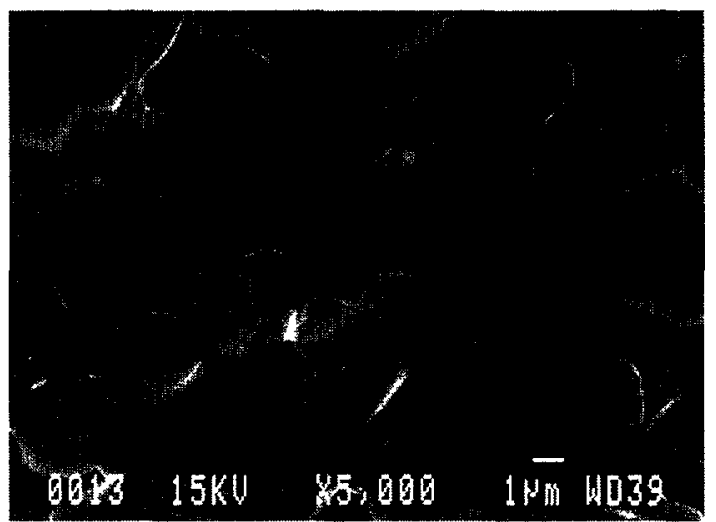

(e)

Fig. 2. - Microstructures des céramiques (a), (b) et (e) obtenues par microscopie électronique à balayage.

[Scanning electronic micrographs of (a), (b) and (e) ceramic samples.]

Tableau II. - Variation de la microstructure avec le type de traitement thermique. [Variation of the ceramic microstructure with annealing conditions.]

\begin{tabular}{|c|l|}
\hline Type de céramique & \multicolumn{1}{c|}{ Microstructure } \\
\hline (a) & $\begin{array}{l}\text { - Taille de grains assez homogène }(\phi=5 \mu \mathrm{m}) \\
\text { - Quelques pores intragranulaires }\end{array}$ \\
(b) & $\begin{array}{l}\text { - Taille de grains homogène }(\phi=1 \grave{a ̀ ~} 2 \mu \mathrm{m}) \\
\text { - Porosité intergranulaire }\end{array}$ \\
(e) & - Taille de grains homogène $(\phi>5 \mu \mathrm{m})$ \\
& - porosité intergranulaire \\
\hline
\end{tabular}




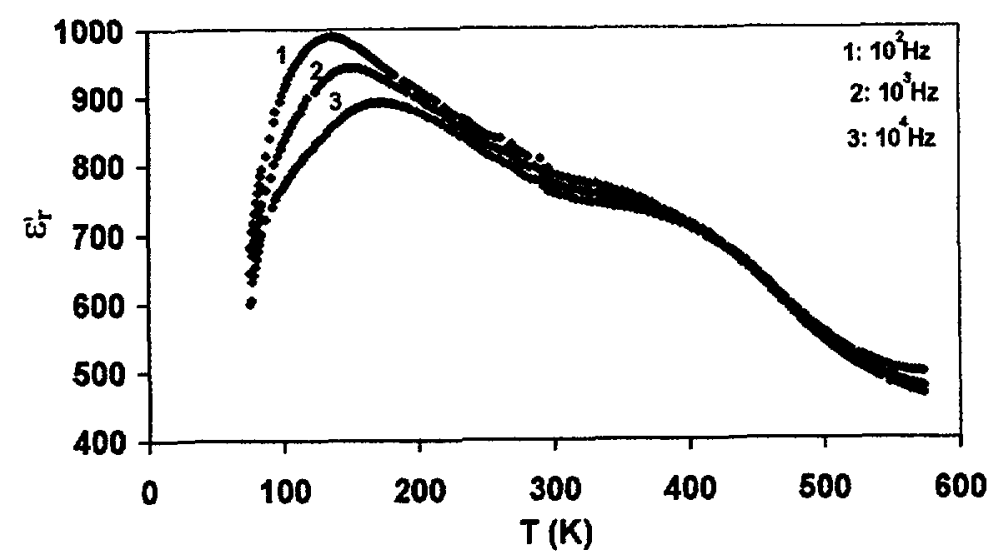

Fig. 3. - Variations thermiques de $\varepsilon_{\mathrm{r}}^{\prime}$ pour la céramique (a).

[Temperature dependence of the permittivity $\varepsilon_{\mathrm{x}}^{\prime}$ for an (a)-type ceramic sample.]

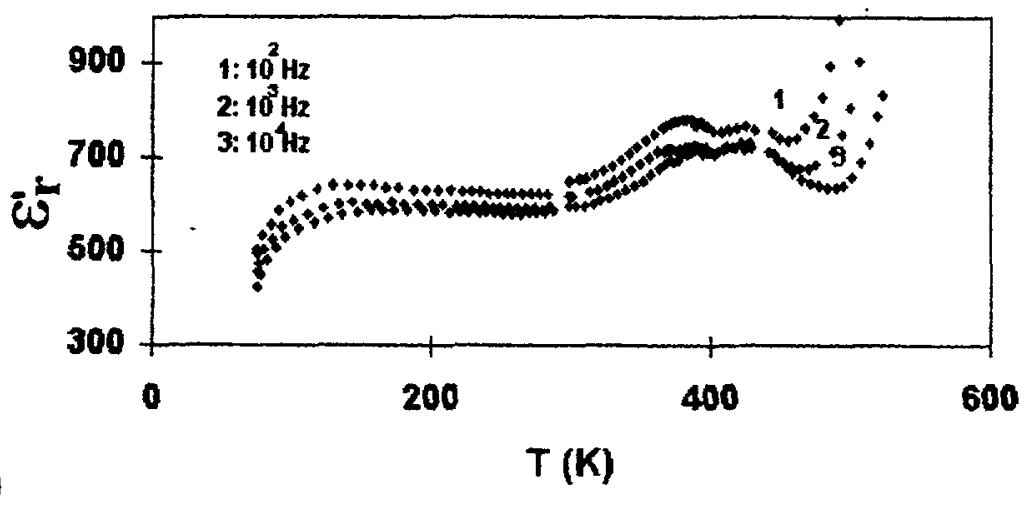

(b)

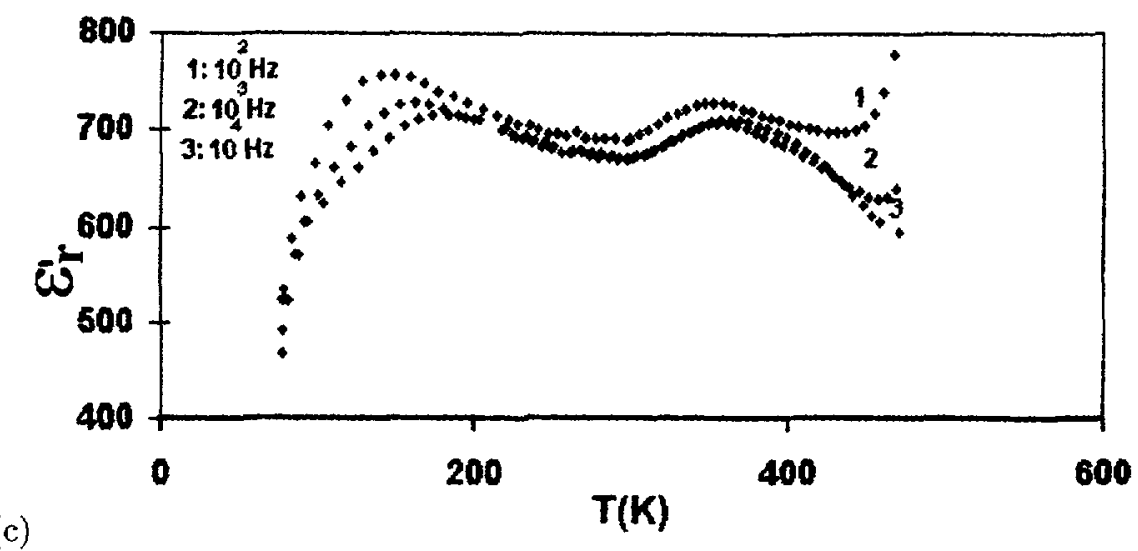

Fig. 4. - Variations thermiques de $\varepsilon_{\mathrm{r}}^{\prime}$ pour les céramiques (b), (c), (d) et (e).

[Temperature dependence of the permittivity $\varepsilon_{\mathrm{r}}^{\prime}$ for (b), (c), (d) and (e) type ceramic samples.] 

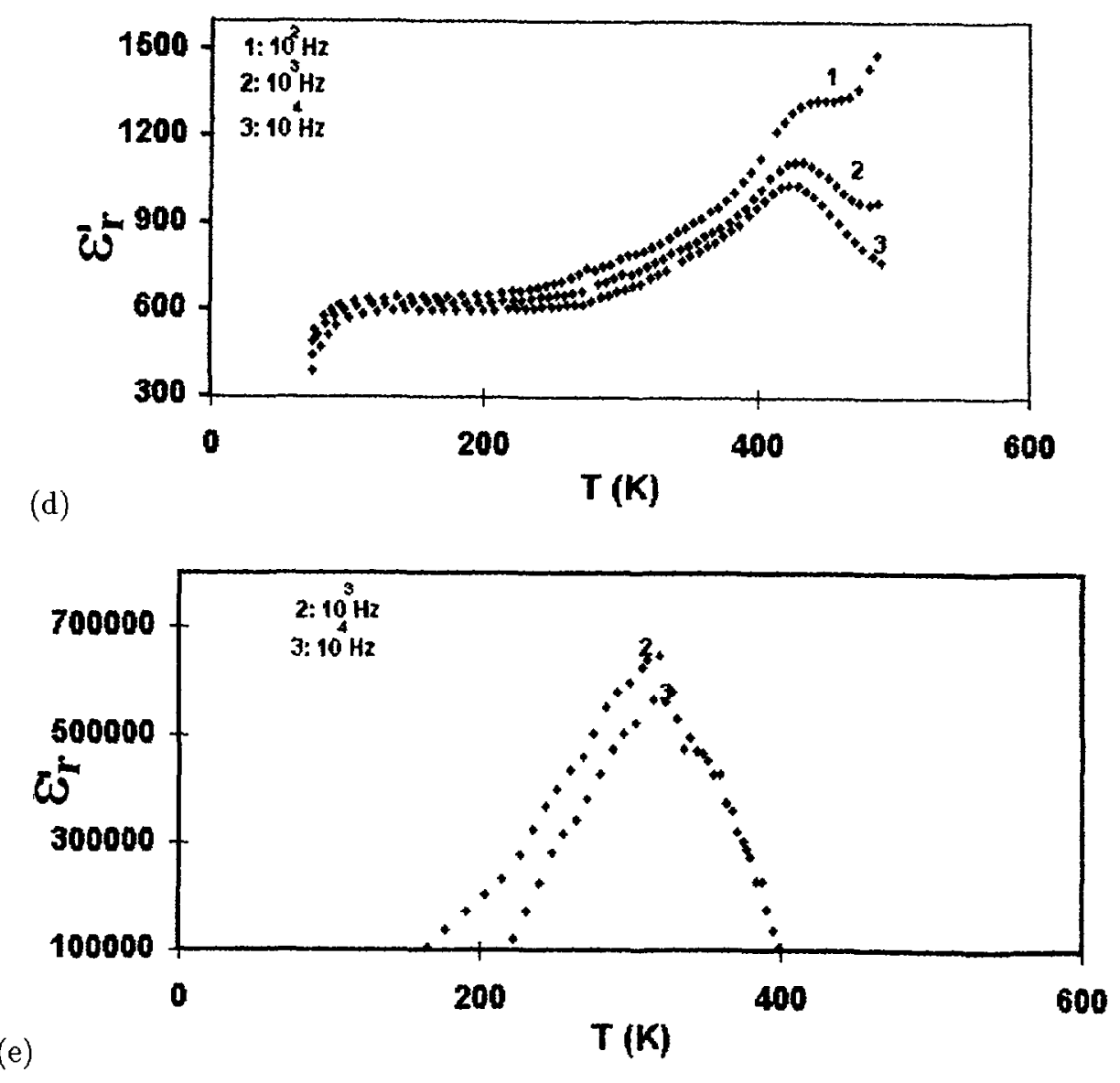

Fig. 4. - (Suite.)

Tableau III. - Variation de la température de Curie avec le type de traitement thermique. [Variation of the Curie temperature with annealing conditions.]

\begin{tabular}{|c|c|}
\hline Type de céramique & $\begin{array}{c}T_{\mathrm{C}}( \pm 5 \mathrm{~K}) \\
\left(f=10^{2} \mathrm{~Hz}\right)\end{array}$ \\
\hline (d) & 430 \\
$(\mathrm{a})$ & 400 \\
(c) & 360 \\
$(\mathrm{e})$ & 320 \\
\hline
\end{tabular}

\section{Mesures de la conductivité électrique}

Des mesures de conductivité électrique ont été effectuées en courant continu, sous hélium (1 atm) entre 100 et $700 \mathrm{~K}$ sur la céramique (e), à l'aide de la méthode des quatre pointes alignées [6]. La figure 5 donnant la variation du logarithme de la conductivité avec l'inverse de la température, montre que le comportement est de type semi-conducteur. 


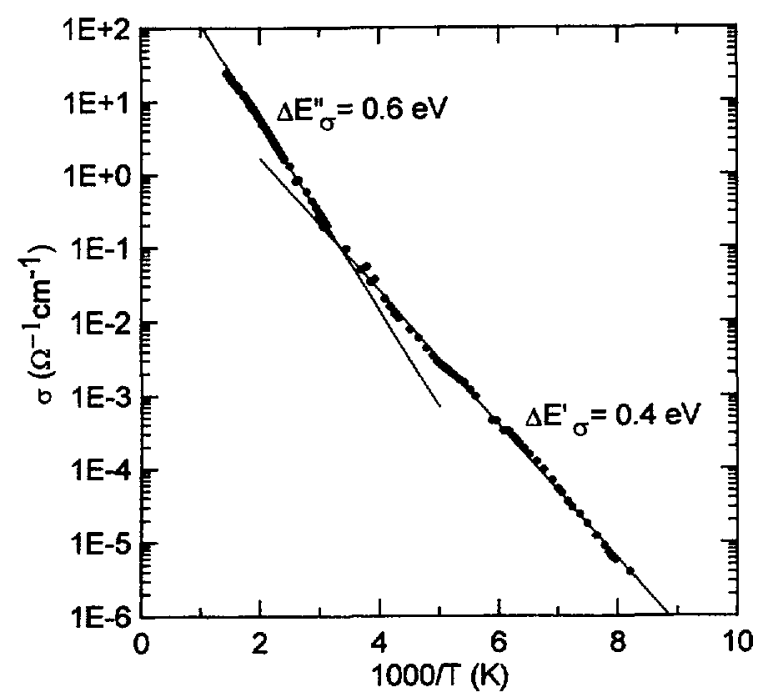

Fig. 5. - Variation du logarithme de la conductivité électrique en fonction de l'inverse de la température.

[Plot of $\log \sigma$ versus $1 / T$ for (e) ceramic sample.]

\section{Pouvoir thermoélectrique}

Le coefficient Seebeck $\alpha$ de la céramique (e) a été mesuré sous hélium entre 160 (température en deçà de laquelle l'impédance de l'échantillon n'est plus adaptée au dispositif de mesure utilisé) et $300 \mathrm{~K}$ à l'aide d'un appareillage décrit par ailleurs [7]. Les résultats obtenus sont donnés à la figure 6 ; $\alpha$ est négatif dans le domaine de température exploré. Ce résultat montre que les porteurs majoritaires sont des électrons. La variation thermique de $\alpha$ confirme le caractère semi-conducteur des échantillons.

\section{Discussion}

Une variation des paramètres de la maille et du système cristallin a été observée uniquement au niveau des échantillons subissant une forte réduction. Toutes les céramiques élaborées présentent, à haute température, une transition ferroélectrique-paraélectrique correspondant à une anomalie ou à un maximum de $\varepsilon_{\mathrm{r}}^{\prime}$ associé à un minimum de $\tan \delta$. En revanche, la courbe de la variation thermique de la céramique (a) fait apparaître seulement un changement de pente. Le pic correspondant à la transition ferroélectrique-paraélectrique est masqué par un effet de relaxation de charges d'espace à basse température. Les mesures diélectriques semblent donc corrélées au type de traitement thermique ainsi réalisé (Figs. 3 et 4 ).

Les matériaux frittés sous oxygène et soumis à un long recuit à $600^{\circ} \mathrm{C}$ sous oxygène présentent une meilleure homogénéité qui se traduit par un pic de $\varepsilon_{\mathrm{r}}^{\prime}$ en température beaucoup plus net que ceux des échantillons subissant un recuit à des températures supérieures à $600^{\circ} \mathrm{C}$. Le traitement prolongé à "basse" température $\left(600^{\circ} \mathrm{C}\right)$ sous oxygène, favorise l'oxydation du matériau. 


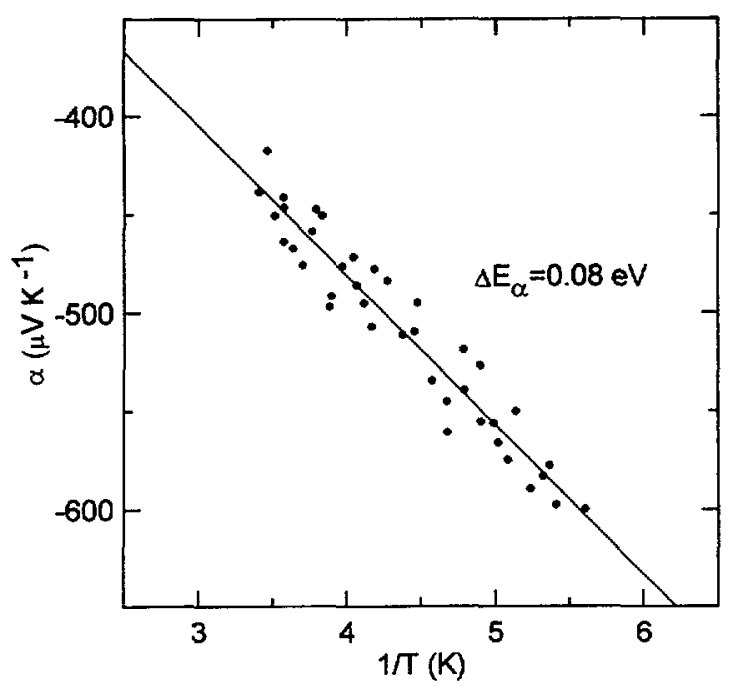

Fig. 6. - Variation thermique du pouvoir thermoélectrique pour la céramique (e). [Temperature dependence of the thermoelectric power versus $1 / T$ for (e) ceramic.]

Le phénomène observé à basse température peut s'expliquer selon les considérations suivantes. Le traitement haute température de la céramique lors du frittage à l'air est générateur de défauts d'oxygène tant dans les grains que dans les joints de grains. Lors du refroidissement, la périphérie des grains aura tendance à se réoxyder plus rapidement que le cour des grains.

Cette inhomogénéité dans la distribution des lacunes d'oxygène entraîne l'apparition de charges d'espace aux joints de grains ; leur caractère très isolant limite le déplacement des charges. Il en résulte une polarisation importante et par conséquent une permittivité élevée au sein du matériau. Un tel phénomène est appelé relaxation diélectrique par charges d'espace ; il apparaît à basse fréquence. Cet effet de relaxation diminue naturellement lorsque les céramiques comportent à la fois des grains et des joints de grains fortement isolants. Cette relaxation disparaît également par un traitement en atmosphère réductrice, les grains et les joints de grains deviennent alors semi-conducteurs et les charges peuvent ainsi se déplacer d'un grain à l'autre.

La composition chimique semble jouer un rôle déterminant en particulier sur l'évolution de la température de Curie. Dans cet esprit, nous allons tenter d'expliquer ces différentes évolutions en tenant compte de l'effet stérique. Comme dans le cas des composés de structure perovskite, le déplacement $\Delta z$ de l'ion central $(\mathrm{Nb}, \mathrm{Ti})$ hors du centre de gravité de l'octaèdre est d'autant plus difficile que sa taille est grande. Selon la relation empirique $T_{\mathrm{C}}(\mathrm{K})=2,00 \times 10^{4}(\Delta z)^{2}(\AA)$ [8], la diminution de $\Delta z$ doit entraîner une diminution de la température de Curie. D'autre part la non-stœchiométrie anionique peut conduire à une coordinence tétraédrique ou pentaédrique pour les atomes de titane ou de niobium, ce qui limiterait le déplacement des cations chargés par suite du volume plus réduit d'un tel environnement oxygéné. Cette diminution du déplacement $\Delta z$ entraînerait une décroissance à la fois de la polarisation spontanée et de la température de Curie. C'est donc bien l'augmentation de la taille des cations $\mathrm{Nb}^{\mathrm{n}+}$ et $\mathrm{Ti}^{\mathrm{n}+}\left(r_{\mathrm{Nb}}^{4+}>r_{\mathrm{Nb}}^{5+}\right.$ et $r_{\mathrm{Ti}}^{3+}>r_{\mathrm{T}_{\mathrm{i}}}^{4+}$ ) et la diminution de coordinence qui sont à l'origine de ce comportement.

L'examen de la figure 5 montre que la conductivité électrique est thermiquement activée. La variation du logarithme de la conductivité avec l'inverse de la température comporte deux domaines linéaires caractérisés par des énergies d'activation $\Delta E_{\sigma}^{\prime}$ à basse température 
$(T<300 \mathrm{~K})$ et $\Delta E_{\sigma}^{\prime \prime}$ à plus haute température $(T>300 \mathrm{~K})$ égales respectivement à 0,4 et $0,6 \mathrm{eV}$. Ces valeurs révèlent un comportement de type semi-conducteur extrinsèque : en effet la largeur de la bande interdite de ces matériaux a une valeur beaucoup plus élevée, de l'ordre de 2,5 à 3,5 eV, comme le suggère d'ailleurs leur aspect incolore.

La figure 6 montre que la céramique (e) obéit à la relation : $\alpha=-k / \mathrm{e}\left(\Delta E_{\alpha} / k T+A\right)$, attendue pour un semi-conducteur. La valeur de l'énergie d'activation $\Delta E_{\alpha}=0,08 \mathrm{eV}$ est très inférieure à celle trouvée pour la conductivité électrique (Fig. 5). Elle correspond à l'énergie d'ionisation des donneurs dans la mesure où les ions $\mathrm{Nb}^{4+}$ (ou les ions $\mathrm{Ti}^{3+}$ ) vont se piéger au voisinage des lacunes d'oxygène pour minimiser l'énergie coulombienne. La différence entre $\Delta E_{\sigma}^{\prime}$ ou $\Delta E_{\sigma}^{\prime \prime}$ et $\Delta E_{\alpha}$ peut être attribuée à une activation thermique de la mobilité des porteurs [9]. On peut remarquer que le changement de pente de la courbe $\log \sigma=f(1 / T)$ intervient à une température très voisine de la température de Curie. Ce résultat n'est pas surprenant dans la mesure où le processus de "hopping" des porteurs est assisté par les phonons et où les phases ferroélectrique et paraélectrique ont naturellement des spectres de phonons différents.

\section{Conclusions}

Des céramiques denses de formule $\mathrm{Sr}_{6} \mathrm{Ti}_{2} \mathrm{Nb}_{8} \mathrm{O}_{30}$ ont été soumises à des traitements thermiques en atmosphère oxydante ou réductrice. Leurs diffractogrammes s'indexent à $300 \mathrm{~K}$ dans le système quadratique ou orthorhombique. L'analyse par microscopie électronique à balayage montre que la taille des grains est assez homogène et varie de 1 à $5 \mu \mathrm{m}$.

Toutes les céramiques élaborées présentent une transition ferroélectrique-paraélectrique. Un phénomène de relaxation diélectrique de charges d'espace apparaît à basse température. Il disparaît par recuit soit sous oxygène, soit en atmosphère réductrice. Ces résultats ont été interprétés par des considérations sur la cinétique des processus d'oxydation et de réduction affectant plus ou moins la périphérie ou le cœur des grains.

Les mesures de conductivité électrique et de pouvoir thermoélectrique effectuées sur l'échantillon le plus réduit révèlent un comportement de semi-conducteur extrinsèque de type $\mathrm{n}$. La comparaison des énergies d'activation suggère que les porteurs ont un caractère polaronique. L'ensemble des résultats obtenus montre l'influence extrêmement importante des traitements thermiques sur les propriétés diélectriques des céramiques de $\mathrm{Sr}_{6} \mathrm{Ti}_{2} \mathrm{Nb}_{8} \mathrm{O}_{30}$.

\section{Bibliographie}

[1] Neurgaonkar R.R., Nelson J.G. and Oliver J.R., Ferroelectric properties of tungsten bronze $\mathrm{M}_{6}^{2+} \mathrm{M}_{2}^{4+} \mathrm{Nb}_{8} \mathrm{O}_{30}$ solid solution systems, Mat. Res. Bull. 27 (1992) 677.

[2] Ainger F.W., Bickley W.P. and Smith G.V., The search for new ferroelectrics with the tungsten bronze structure, Proc. Brit. Ceram. Soc. 18 (1970) 221.

[3] Kryshtop V.G., Devlikanova R.U. and Fesenko E.G., Synthesis and X-Ray diffraction study of compounds with the tetragonal tungsten bronze structure, Izv. Akad. Nauk. Neorg. Mater. 15 (1979) 1777.

[4] Ikeda T., Haraguchi T., Onodera V. and Saito T., Some compounds of tungsten-bronze type $\mathrm{A}_{6} \mathrm{~B}_{10} \mathrm{O}_{30}(\mathrm{~B}=\mathrm{Nb}$, Ti or Nb,W), Jap. J. Appl. Phys. 10 (1971) 987.

[5] Stephenson N.C., Crystal structure of $\mathrm{Ba}_{3}\left(\mathrm{TiNb}_{4}\right) \mathrm{O}_{15}$, Acta. Cryst. 18 (1965) 496. 
[6] Laplume J., Bases théoriques de la mesure de la résistivité et de la constante de Hall par la méthode des pointes, Onde Electrique 35 (1955) 355.

[7] Dordor P., Marquestaut E. et Villeneuve G., Dispositif de mesures du pouvoir thermoélectrique sur des échantillons très résistants entre 4 et $300 \mathrm{~K}$, Rev. Phys. Appl. 15 (1980) 1670.

[8] Abrahams S.C., Kurtz S.K. and Jamieson P.B., Atomic displacement relationship to Curie temperature and spontaneous polarization in displacive ferroelectrics, Phys. Rev. 172 (1968) 551.

[9] Mott N.F., "Metal-Insultor Transitions", Taylor and Francis, Eds. (London, 1990) p. 59. 